\title{
A COMPARATIVE STUDY ON ADAPTIVE LIFTING BASED SCHEME AND INTERACTIVE ARTIFICIAL BEE COLONY ALGORITHM FOR IMAGE COMPRESSION
}

\author{
G.G Rajput ${ }^{1}$ and Vrinda Shivashetty ${ }^{2}$ \\ ${ }^{1}$ Department of Computer Science, Rani Channamma University, Belagavi, India \\ ${ }^{2}$ Department of Computer Science, Gulbarga University Gulbarga, India
}

\begin{abstract}
Adaptive update lifting scheme based Interactive artificial bee colony algorithm is proposed in this paper. Wavelet transform based compression technique is used for images and multimedia files. Approximation and detail coefficients are extracted from the signal by filtering in wavelet transform. To increase frequency resolution both approximation and detail coefficients are re-decomposed up to some level. Artificial bee colony algorithm by local search finds different update coefficients to get quality of compressed image by choosing optimally best update coefficient. In IABC, the affection between employed bees and the onlooker bees is found by considering the concept of universal gravitation. By passing on control parameter different values, the universal gravitation involved in the IABC has a single onlooker bee \& variety of quantities of employed bees. As a result, IABC compared with existing image compression schemes such as wavelet transform and Artificial Bee colony Algorithm, the proposed work gives better PSNR.
\end{abstract}

\section{KEYWORDS}

Image Compression, Artificial bee colony, Wavelet Transform, Adaptive Lifting Scheme.

\section{INTRODUCTION}

Wavelet coding is recognised as efficient coding technique for lossy image compression. It is well known that, the wavelet transform decomposes a typical image data to a few coefficients with large magnitude and many coefficients with small magnitude. As most of the energy of the image concentrates on these coefficients with large magnitude, lossy compression systems just by using coefficients with large magnitude can realize the reconstructed image with good quality and high compression ratio. For wavelet transforms, Lifting Scheme(LS) (3,4)allows efficient construction of the filter banks. The restriction of this structure is that the filter structure is fixed over the entire signal. In many applications to shape itself to the signal it is very much desirable to design the filter banks. A number of such adaptive Lifting Schemes are proposed in the literature which consider local characteristics of the signal for adapting $[13,16]$. Ramanathan et al.[15] applied ABC algorithm to determine the optimal window size to obtain satisfactory compression and quality in the multi-objective manner. M. Mohamed Ismail and Dr. K.Baskaran 
The International Journal of Multimedia \& Its Applications (IJMA) Vol.7, No.2, April 2015

[16] have implemented $\mathrm{ABC}$ algorithm in update process of lifting scheme to get better PSNR. PEI-WEI TSAI et al[23] have proposed the Interactive Artificial Bee Colony Optimization algorithm.

Image compression technique using adaptive update lifting scheme and Interactive Artificial Bee Colony Algorithm (IABC) is presented in this paper. The results obtained are compared with wavelet transform \& Artificial Bee Colony Algorithm proposed in the literature. The rest of the paper is described as follows. Section II provides a brief overview of basic image compression techniques. In section III, a general lifting scheme is discussed and compared with the adaptive lifting scheme. Section IV discusses about the wavelet transform. In Section V, Artificial Bee Colony Algorithm is explained and section VI describes the Interactive Artificial Bee Colony algorithm and Experimental results are discussed in Section VII.

\section{BASIC IMAGE COMPRESSION TECHNIQUES}

The image compression techniques are generally classified into two categories depending whether or not an exact replica of the original image could be reconstructed using the compressed image. These are:

\section{Lossy Image Compression technique \\ 2. Lossless Image Compression technique}

\section{Lossy Image Compression Techniques}

Lossy schemes provide much higher compression ratios than lossless schemes. Lossy schemes are widely used since the quality of the reconstructed images is adequate for most applications. By this scheme, the decompressed image is not identical to the original image, but reasonably close to it. The most popular current lossy image compression methods use a transform-based scheme[25].

\section{Lossless Image Compression Techniques}

In lossless compression techniques, the original image can be perfectly recovered from the compressed image. These are also called noiseless since they do not add noise to the signal. It is also known as entropy coding since it use decomposition techniques to minimize redundancy[21].

\section{LIFTING SCHEME}

Lifting scheme is simplest and efficient algorithm to calculate wavelet transforms. Lifting scheme is used to implement critically sampled filter banks which have integer output. The lifting scheme can custom design the filters, essential in the transform algorithms. Independent of translating and dilating, needless of frequency analysis, lifting scheme is processed into space domain. An answer to the algebraic stage of wavelet construction is provided by lifting scheme, which leads to a fast in-place calculation of the wavelet transform, i.e. it does not require auxiliary memory. Different wavelets show different image compression effect; the compressed image quality and the compression rate are not only relational to the filter length, but also concerns with regularity and local frequency, vanishing moment, orthogonality, bi-orthogonality. In this paper, we 
The International Journal of Multimedia \& Its Applications (IJMA) Vol.7, No.2, April 2015

implement adaptive lifting scheme based upon wavelet decomposition. Then, with the help of IABC algorithm, we find the best directional window size to get better compression ratio with considerable quality.

\section{A. The Lifting Concept}

Lifting is a spatial (or time) domain construction of bi-orthogonal wavelets. The lifting scheme procedure consists of three steps: Split, Predict and Update (Fig. 1) and inverse Lifting scheme is shown in Fig. 2.

\section{Split:}

Split the original data into two disjoint subsets. Though any disjoint split is possible, in the standard lifting scheme we split the original data set $x[n]$ into the even indexed points, $x e[n]-$ $x[2 n]$, and the odd indexed points $x o[n]=x[2 n+1]$

\section{Predict:}

Generate the detail signals $\mathrm{d}[\mathrm{n}]$ as the predicting error using prediction operator $\mathrm{P}$

\section{Update:}

To obtain scaling coefficients $\mathrm{c}[\mathrm{n}]$ that represent a coarse approximation to the original signal $\mathrm{x}[\mathrm{n}]$ merge $\mathrm{xe}[\mathrm{n}]$ and $\mathrm{d}[\mathrm{n}]$. This is accomplished by applying an $\mathrm{U}$ update operator to the wavelet coefficients and adding to

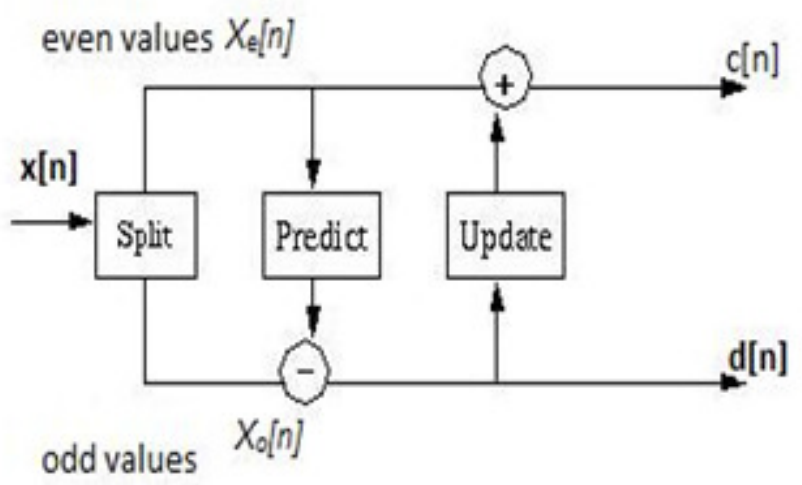

Figure 1. Lifting stage: Split, Predict, Update

As long as for the inverse and forward transforms $\mathrm{U}$ and $\mathrm{P}$ are chosen, the original signal will be perfectly reconstructed. The inverse lifting stage is shown in Fig.2. 
The International Journal of Multimedia \& Its Applications (IJMA) Vol.7, No.2, April 2015

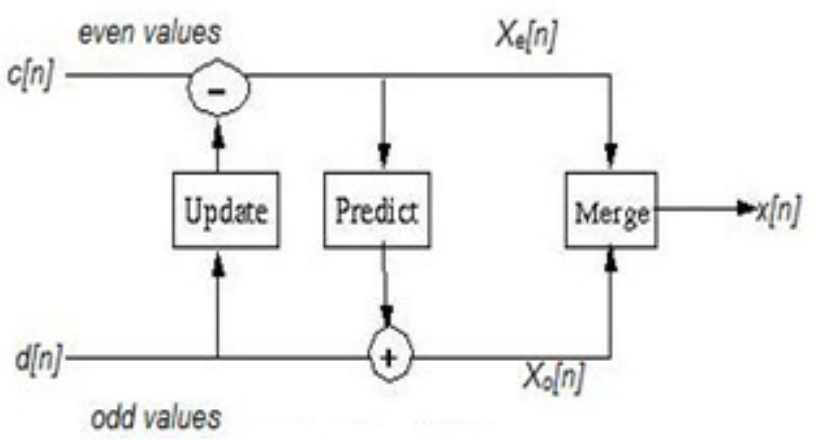

Figure 2. Inverse lifting steps: undo the Update, undo the Predict, and Merge the even and odd samples

\section{B. Adaptive lifting scheme}

The adaptive lifting scheme is classical lifting modification. Fig.3 shows the adaptive update lifting scheme followed by a fixed prediction. At each sample n, according to a decision function $\mathrm{D}(\mathrm{x}[\mathrm{n}], \mathrm{y})$ an update operator is chosen. As in the classical and space-varying lifting, the critical point is that $\mathrm{D}(\mathrm{x}[\mathrm{n}], \mathrm{y})$ depends on $\mathrm{y}$, and it also depends on the sample being updated. The update operator and addition are fixed, in the standard lifting scheme. The choice of addition and the update operator depends on the information locally available within both the approximation signal and the detail signal in the adaptive lifting scheme.

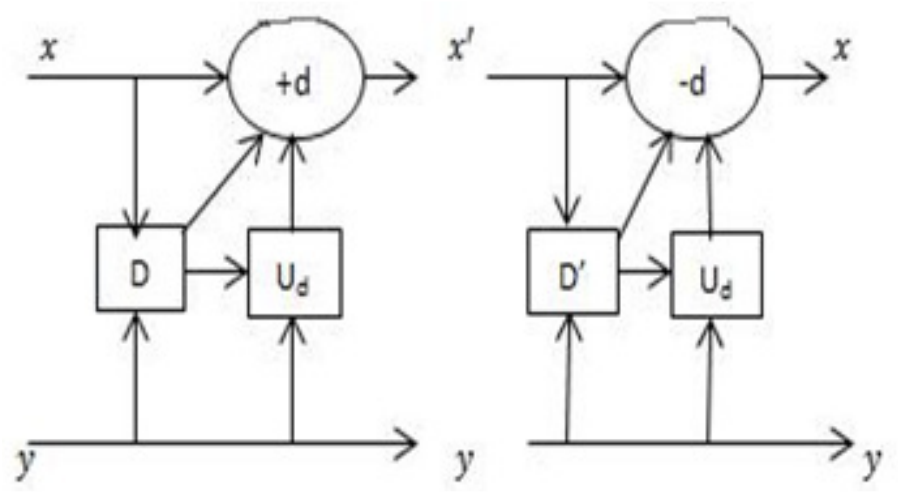

Figure 3. Adaptive update lifting scheme

According to the structure of lifting, Adaptive Lifting Scheme performs update first, and then performs prediction.

\section{WAVELET TRANSFORM}

The wavelet transform decomposes a typical image data to a few coefficients with large magnitude and many coefficients with small magnitude. In wavelet transform first step decomposes a signal into constituent parts in the time-frequency domain on a basis function localized in both time and frequency domains. The image or signal is decomposed into four different frequencies: approximation, vertical detail, horizontal detail and diagonal detail. 
The International Journal of Multimedia \& Its Applications (IJMA) Vol.7, No.2, April 2015

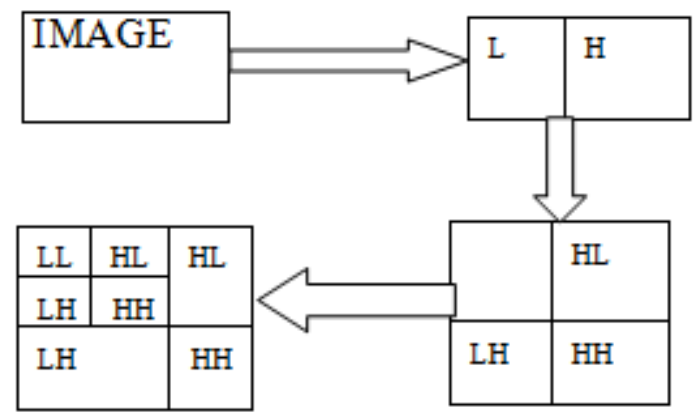

Figure 4. 2-level Wavelet decomposition

Up to a level the decompositions are repeated on the approximation coefficients. As details are not decomposed at the high levels and can be described by the small scale wavelet coefficients, wavelet transform is not suitable for images having rapid variations.

\section{ARTificial BEE COLONY AlgorithM (ABC)}

Karaboga [17] in 2005 proposed $\mathrm{ABC}$ algorithm and the performance of $\mathrm{ABC}$ is analyzed in 2007 [18]. The ABC algorithm is developed by inspecting the behaviours of the bees on finding food source, called as nectar, and the information of food sources to the bees in the nest is shared. The different phases of $\mathrm{ABC}$ are classified into three types, namely, the employed bee, onlooker bee, and the scout bee. In the employed bee phase, the employed bee stays on a food source and provides the neighbourhood of the source in its memory. In the onlooker phase, the onlooker receives the information of food sources from the employed bees in the hive and to gather the nectar one of the food source is selected; and for finding new food, the new nectar, sources the scout is responsible. The process of the $\mathrm{ABC}$ algorithm is presented as follows:

Step 1. Initialization: In a solution space randomly Spray ne percentage of the populations, and then their fitness values calculated, called as nectar amounts, where the ratio of employed bees to the total population is represented by ne. The populations positioned into the solution space, are called as employed bees.

Step 2. Move the Onlookers: The probability of selecting a food source is calculated, select a food source to move by roulette wheel selection for each onlooker bees and then determine the nectar amounts of them.

Step 3. Move the Scouts: By a continuous predetermined number of iterations, if the fitness values of the employed bees do not improve, which is called "Limit", such food sources are abandoned, and these employed bees become the scouts. The movement of scouts takes place.

Step 4. Update the Best Food Source Found So Far: Memorize the position and the best fitness value, which are found by the bees.

Step 5. Termination Checking: Check whether the termination condition is satisfied by the number of iterations. Terminate the program and output the results if the termination condition is satisfied; otherwise go back to the Step 2. 
The International Journal of Multimedia \& Its Applications (IJMA) Vol.7, No.2, April 2015

\section{INTERACTIVE ARTIFICIAL BEE COLONY ALGORITHM (IABC)}

In IABC algorithm, in the solution space randomly spray $n_{e}$ percentage of the populations , then fitness values called as nectar amounts is calculated, which represents the ratio of employed bees to the total population. When these populations are positioned into the solution space they are called employee bees. The probability of selecting a food source is then calculated. Select a food source to move by roulette wheel selection for every onlooker bees and then nectar amounts of them is determined. If the employed bees fitness values does not improve by predetermined number of iterations continuously, called "Limit", such food sources are abandoned, and these employed bees become the scouts. The scouts are moved. The position and the best fitness value found by the bees are memorized. We check whether the termination condition is satisfied by the total number of iterations. If the condition for termination is satisfied, terminate the program and output the results. The flow chart for IABC is shown in Figure 5.

In the proposed method, the input image is decomposed using wavelet lifting scheme and then the Interactive artificial bee colony algorithm is used in the update process to get considerable quality.

\section{A. Algorithm}

Step 1: Input the Image.

Step 2: Split the image into odd and even pixel regions.

Step 3: Decompose the image as (odd-even) for next prediction step.

Step 4: Fix the maximum coverage size as ' $M$ ' and initialized ' $K=0$ ' for prediction co-efficient. Where $\mathrm{M}$ is maximum window size, upto which it will do local search for each center pixels maximum window size in our program is 5 .

Step 5: Each pixel in the decomposed image is Scanned and its present fitness value and compression ratio is calculated.

Step 6: To predict 'a' and 'b' call direction finding algorithm co-efficient of all 8-direction combination. 
The International Journal of Multimedia \& Its Applications (IJMA) Vol.7, No.2, April 2015

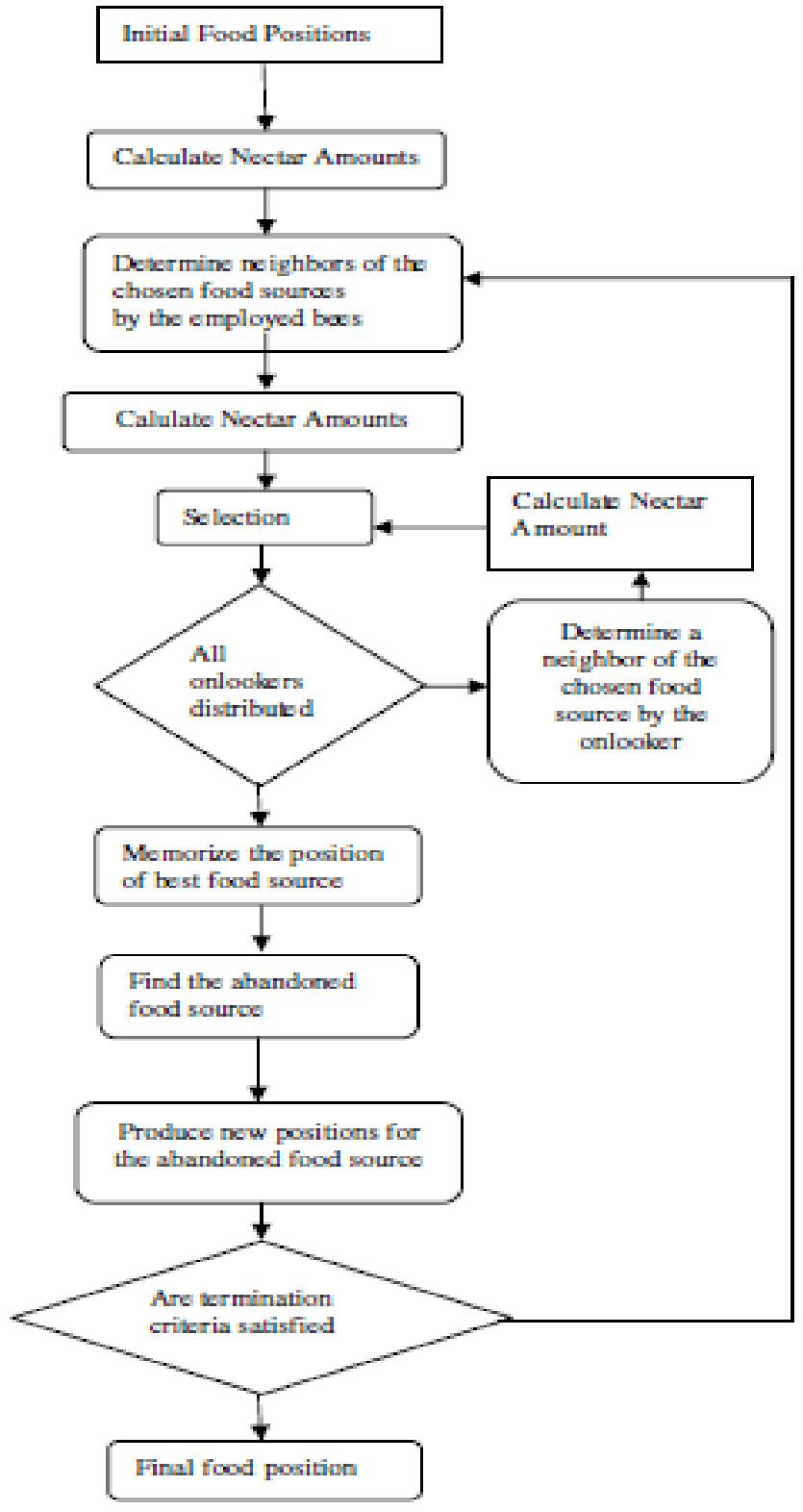

Figure 5. Flow chart for IABC Algorithm

\begin{tabular}{|l|l|l|l|l|l|}
\hline & DL & & VT & DR & \\
\hline & & DL & VT & & \\
\hline HL & HL & HL & X & HR & HR \\
\hline & DD & DD & VD & DB & \\
\hline DD & & & VD & & DB \\
\hline
\end{tabular}

Figure 6. Directional coefficient for center pixel ' $\mathrm{x}$ ' 
The International Journal of Multimedia \& Its Applications (IJMA) Vol.7, No.2, April 2015

Where $\mathrm{x}$ is an center pixel to be update

HL is predicted coefficient in horizontal left direction

HR is predicted coefficient in horizontal right direction

VT is predicted coefficient in vertically top direction

VD is predicted coefficient in vertically down direction

DL is predicted coefficient in diagonally top left direction

DR is predicted coefficient in diagonally top right direction

DB is predicted coefficient in diagonally bottom left direction

DD is predicted coefficient in diagonally bottom right direction

Step 7: By using Update lifting formula for each direction prediction calculate update weight and find compression ratio and PSNR.

Step 8: The best individual is memorized, CR and its direction using IABC local search.

Step 9: To predict and update the best value for different range of window size iterate $\mathrm{K}$ from (0 to $\mathrm{M})$.

Step 10: Using IABC local search, memorize the best window size in terms of its MSE and CR for each reference pixel.

\section{EXPERIMENTAL RESULTS}

The IABC algorithm is tested on standard images with different image formats such as tif, png jpeg. The results obtained using IABC are shown in figure 7. The results are tabulated for various images in Table(1) \& Table(2). Figure 8 \& Figure 9 respectively presents the result for Cameraman Image \& Pepper Image graphically. The results obtained are with the results of different image compression scheme such as wavelet transform, Artificial Bee colony algorithm $\&$ Interactive Artificial Bee colony algorithm.

Image 1: Cameraman Image
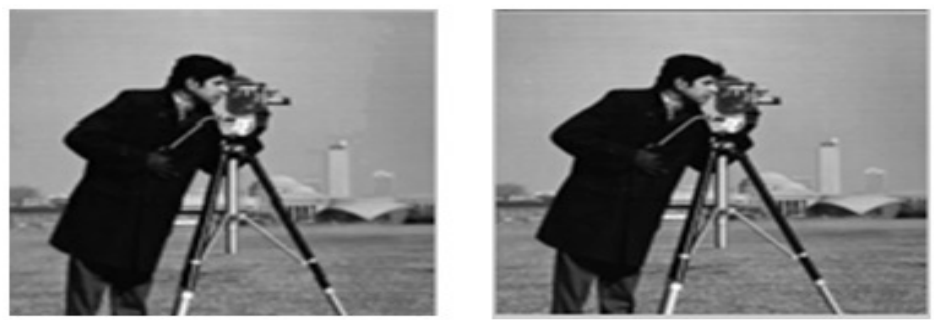

Image 2: Peppers Image

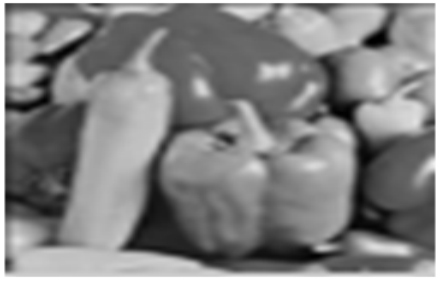

(a)

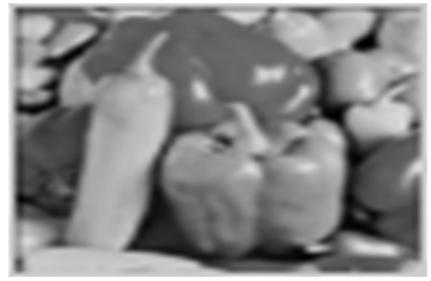

(b)

Figure 7. Reconstructed Images with Lifting using IABC (a) Original Image (b) Output Image. 
The International Journal of Multimedia \& Its Applications (IJMA) Vol.7, No.2, April 2015

\section{A. Comparison Tables}

Table 1: Comparison table for cameraman image

\begin{tabular}{|c|c|c|c|}
\hline $\begin{array}{l}\text { Sl.No. } \\
\text { [Ref. No.] }\end{array}$ & Referred Method & $\begin{array}{l}\text { Compression } \\
\text { Ratio }\end{array}$ & PSNR \\
\hline \multirow[t]{4}{*}{$1[16]$} & \multirow{4}{*}{$\begin{array}{l}\text { With Wavelet } \\
\text { Transform }\end{array}$} & 30 & 33.135 \\
\hline & & 40 & 29.635 \\
\hline & & 50 & 27.135 \\
\hline & & 60 & 25.635 \\
\hline \multirow[t]{4}{*}{$2[16]$} & \multirow{4}{*}{$\begin{array}{l}\text { With Lifting Scheme } \\
\text { using ABC }\end{array}$} & 30 & 40.52 \\
\hline & & 40 & 38.11 \\
\hline & & 50 & 36.03 \\
\hline & & 60 & 35.86 \\
\hline \multirow[t]{4}{*}{$3[24]$} & \multirow{4}{*}{$\begin{array}{l}\text { Proposed Method } \\
\text { [Lifting Scheme using } \\
\text { Interactive ABC] }\end{array}$} & 30 & 41.78 \\
\hline & & 40 & 38.92 \\
\hline & & 50 & 38.63 \\
\hline & & 60 & 34.26 \\
\hline
\end{tabular}

Table 2: Comparison table for Pepper Image

\begin{tabular}{|l|l|l|l|}
\hline Sl.No. \\
[Ref.No.] & Referred Method & $\begin{array}{l}\text { Compression } \\
\text { Ratio }\end{array}$ & PSNR \\
\hline \multirow{3}{*}{$1[16]$} & \multirow{2}{*}{$\begin{array}{l}\text { With Wavelet } \\
\text { Transform }\end{array}$} & 30 & \\
\cline { 3 - 4 } & & 40 & 27.55 \\
\cline { 3 - 4 } & & 50 & 25.05 \\
\cline { 3 - 4 } & & 60 & 23.55 \\
\hline \multirow{2}{*}[16]{} & With Lifting Scheme & 30 & 20.95 \\
\cline { 3 - 4 } & using ABC & 40 & 38.90 \\
\cline { 3 - 4 } & & 50 & 37.78 \\
\cline { 3 - 4 } & & 60 & 35.97 \\
\hline \multirow{3}{*}{$3[24]$} & Proposed Method & 30 & 34.42 \\
\cline { 3 - 4 } & [Lifting Scheme using & 40 & 30.83 \\
\cline { 3 - 4 } & Interactive ABC] & 50 & 37.85 \\
\cline { 3 - 4 } & & 60 & 34.60 \\
\hline
\end{tabular}


The International Journal of Multimedia \& Its Applications (IJMA) Vol.7, No.2, April 2015

\section{A. Comparison Graphs}

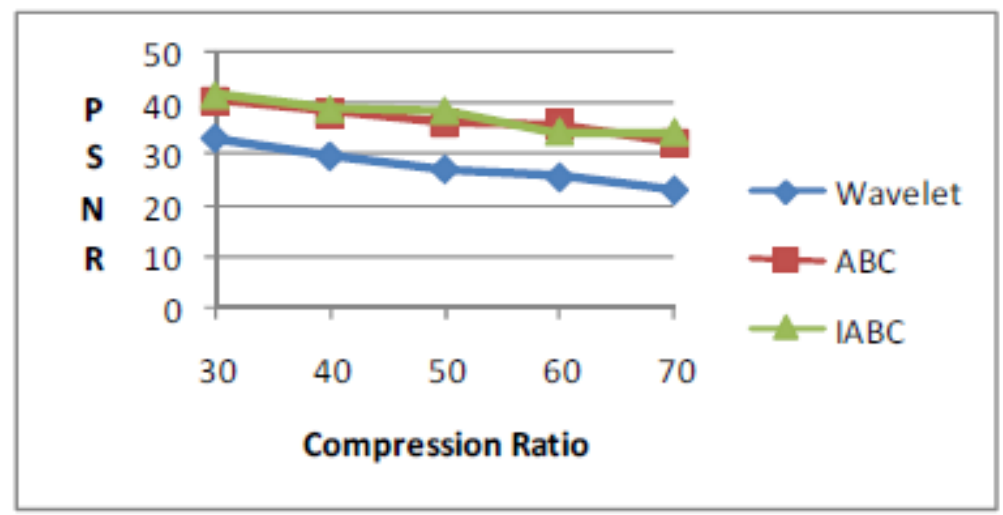

Figure 8. Comparison Graph for Cameraman Image

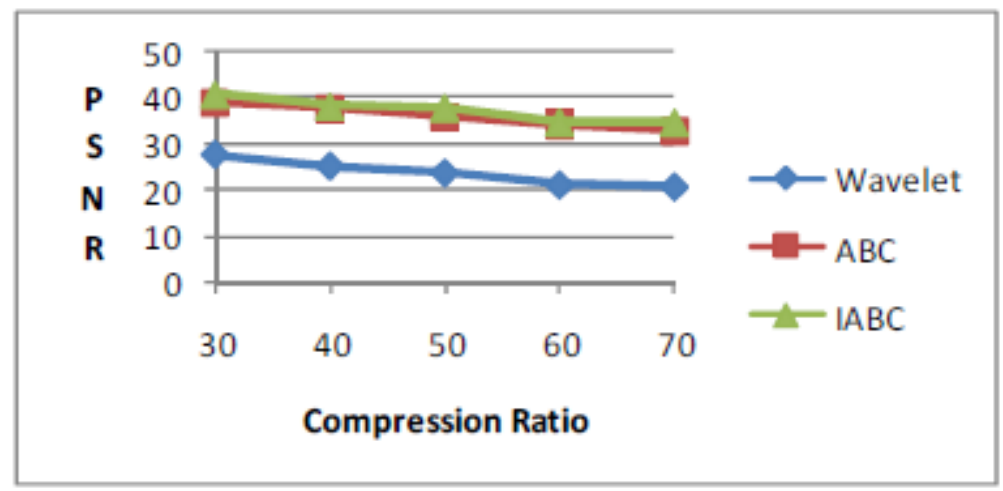

Figure 9. Comparison Graph for Pepper Image

\section{CONCLUSION AND FUTURE ENHANCEMENTS}

In this paper, Interactive Artificial Bee Colony algorithm is implemented in update process of lifting scheme to give better PSNR. From the experimental results, it is concluded that IABC method gives best results compared to other techniques like wavelet and Artificial Bee Colony method. It gives the way to reduces in data to represent the image and decrease in transmission bandwidth. So, the transmission cost and memory cost is reduced with help of IABC method.

In future work, Interactive Artificial Bee Colony algorithm can be implemented in the thresholding process to reduce the number of coefficient representing the image by optimally choosing the thresholding value to get more better compression and quality.

\section{REFERENCES}

[1] Subramanya A. "Image Compression Technique, " potentials IEEE, Vol. 20, issue 1,pp19-23, FebMarch 2001.

[2] Rafael C. Gonzalez, Richard Eugene; “ Digital image processing”, Edition 3, 2008,page 466. 
The International Journal of Multimedia \& Its Applications (IJMA) Vol.7, No.2, April 2015

[3] W. Sweldens, "The lifting scheme: A new philosophy in biorthogonal wavelet constructions", in Proc. SPIE, vol. 2569,1995,pp. 68-79.

[4] W. Sweldens, "The lifting scheme: A construction of second-generation wavelets", SIAM J. Math. Anal., vol. 29, no. 2, pp. 511-546,1997.

[5] A.R. Calderbank, I Daubechies, W. Sweldens, and B-L Yeo, "Wavelet transforms that map integers to integers", J. Appl. Comput. Harmon, Anal., vol.5, no. 3, 1998.

[6] M. Adams and F. Kossentini, "Reversible Integer-to-Integer Wavelet Transforms for Image Compression: Performance Evaluation and Analysis", IEEE Trans.On Image Processing, vol.9, no.6, pp. 1010-1024, Jun 2000.

[7] N.V Boulgouris, D. Tzovaras, and M.G. Strintzis, "Lossless image compression based on optimal prediction, adaptive lifting, and conditional arithmetic coding”, IEEE, Trans. Image Process., vol. 10, no. 1,pp. 1-14, Jan. 2001.

[8] M. Dorigo and L.M. Gambardella, "Ant Colony Optimization for Data Clustering”, Proc. Of 8th Pacific Rim International Conference on Artificial Intelligence, Auckland, New Zealand, LNAI 3157, pp. 534-543,2004.

[9] J. Kennedy, R.C. Eberhart, and Y.Shi, "Swarm Intelligence", Morgan Kaufmann Publishers, San Francisco,2001.

[10] B. Akay and D. Karaboga, "Parameter tuning for the artificial bee colony algorithm," ICCCI 2009 (R.Kowalezyk, N.T. Nguyen and S.M.Chen,eds.), LNAI, vol. 5796, 2009, pp. 608-619.

[11] V . U Kale and N.N. Khalsa, "Performance evaluation of various wavelets for image compression of natural and artificial images", International Journal of Computer Science and communication 1(2010), no.1, pp. 179-184.

[12] D. Karaboga and B. Akay, A survey: "Algorithms simulating bee swarm intelligence", Artificial Intelligence Review 31(2009), no. 1, pp. 55-68.

[13] G. Piella and H.J.A.M. Heijmans, "Adaptive lifting schemes with perfect reconstruction", Research Report PNARO104,CWI, Amsterdam, Feb. 2001.

[14] F.W. Moore, "A genetic algorithm for optimized reconstruction of quantized signals", Evolutionary computation, 2005. The 2005 IEEE congress on, vol. 1,2005, pp. 105-111.

[15] R. Ramanathan, K. Kalaiarasi, D. Prabha, "Improved wavelet based compression with adaptive lifting scheme using Artificial Bee Colony algorithm", International Journal of Advanced Research in Computer Engineering \& Technology, Vol 2 , Issue 4, April 2013.

[16] M. Mohamed Ismail, Dr. K. Baskaran, “Adaptive Lifting Based Image Compression Scheme Using Artificial Bee Colony Algorithm”, International Journal of Electronics Communication and Computer Engineering, Vol. 4, Issue 1, ISSN(print):2278-4209, 2013.

[17] W. Trappe and K.J.R.Liu, "Adaptivity in the lifting scheme," in 33th Conference on Information science and systems, Baltimore, March 1999, pp. 950-958.

[18] D. Karaboga, “ An Idea Based On Honey Bee Swarm For Numerical Optimization”, Technical Report-TR06, Erciyes University, Engineering Faculty, Computer Engineering Department, 2005.

[19] D. Karaboga and B.Basturk. "On the performance of artificial bee colony (ABC) algorithm", Applied Soft Computing 8(2008), pp. 687-697, 2008

[20] E. Bonabeau, M. Dorigo, and G. Theraulaz, "Swarm Intelligence: From Natural to Artificial Intelligence", NY: Oxford University Press, 1999.

[21] Richa Goyal, Jasmeen Jaura, “ A Review of Various Image Compression Techniques”, International Journal of Advanced Research in Computer Science and Software Engineering, Vol. 4, Issue 7, July 2014.

[22] Y. Guo, X.Gao, H. Yin, and Z. Tang, "Coevolutionary Optimization Algorithm with Dynamic Subpopulation Size", International Journal of Innovative Computing, Information and Control, vol.3, no.2, pp.435-448, 2007.

[23] Pei-Wei Tsai, Jeng-Shyang Pan, Bin-Yih Liao, and Shu- Chuan Chu," Enhanced Artificial Bee Colony Optimization", International Journal of Innovative Computing, Information and control, vol.5, no.12, pp.1-12, Dec. 2009. 
The International Journal of Multimedia \& Its Applications (IJMA) Vol.7, No.2, April 2015

[24] Vrinda Shivashetty, G.G Rajput, "Adaptive Lifting Based Image Compression Scheme Using Interactive Artificial Bee Colony Algorithm”, CSITY, SIGPRO, DTMN-2015, pp. 09-21, DOI: $10.5121 /$ csit.2015.50302

[25] Dalijit Singh, Sukhjeet Kaur Ranade, "Comparative Analysis of Transform based Lossy Image Compression Techniques", International Journal of Engineering Research and Applications(IJERA), Vol.2, Issue 5, September-October 2012, pp. 1736-1741.

\section{AUTHORS}

Dr. G. G. Rajput currently working as Associate Professor in the Department of Computer Science at Rani Channamma University Belagavi, Karnataka State, India. The Area of interest includes Image processing, Pattern recognition, Operations Research and Software Engineering.

Vrinda Shiva Shetty received B.E from Gurbarga University and M.Tech degree from VTU in Computer Science and Engineering and presently pursuing Ph.D in Image Compression from the University of Gulbarga University, Gulbarga, and Karnataka. Field of Interest includes Intelligent Image Processing, Evolutionary Computation. 